\title{
New GTC Spectroscopic Data and a Statistical Study to Better Constrain the Redshift of the BL Lac RGB J2243+203
}

D. Rosa González, ${ }^{1 \star}$ H. Muriel, ${ }^{2,3}$ Y. D. Mayya, ${ }^{1}$ I. Aretxaga, ${ }^{1}$ J. Becerra González, ${ }^{4,5}$ A. Carramiñana, ${ }^{1}$ J. Méndez-Abreu, ${ }^{4,5}$ O. Vega, ${ }^{1}$ E. Terlevich, ${ }^{1}$ S. Coutiño de León, ${ }^{1}$ A. Furniss, ${ }^{6}$ A. L. Longinotti, ${ }^{1}$ R. J. Terlevich, ${ }^{1}$ A. C. Pichel, ${ }^{7}$ A. C. Rovero, ${ }^{7}$ and C. Donzelli 2,3

1 Instituto Nacional de Astrofísica, Optica y Electrónica, Tonantzintla, 72840 Puebla, Mexico

2 Instituto de Astronomía Teórica y Experimental (IATE), CONICET-Universidad Nacional de Córdoba, Córdoba, Argentina

3 Observatorio Astronómico, Universidad Nacional de Córdoba, Córdoba, Argentina

4 Instituto de Astrofísica de Canarias, C/ Vía Láctea s/n, E-38205 La Laguna, Spain

5 Departamento de Astrofísica, Universidad de La Laguna, E-38206 La Laguna, Spain

6 Physics Department, California State University East Bay, Hayward, CA 94542, USA

7 Instituto de Astronomía y Fúsica del Espacio (IAFE, CONICET-UBA), Av. Inte. Güiraldes 2620, C1428, ZAA Buenos Aires, Argentina

\section{Accepted XXX. Received YYY; in original form ZZZ}

\begin{abstract}
We present new spectroscopic data of the BL Lac RGB 2243+203, and its surroundings, obtained with the OSIRIS Multi Object Spectrograph (MOS) mounted in the Gran Telescopio Canarias (GTC). The spectra of neither the BL Lac nor its host galaxy show any spectral feature, thus hindering direct determination of its redshift. The spectroscopic redshift distribution of objects in the MOS field of view shows four galaxies with redshift between 0.5258 and 0.5288 . We make use of a statistical analysis to test the possibility that the targeted BL Lac may be a member of that group. By using the spectroscopic redshifts obtained with our GTC observations, we found that this probability is between $86 \%$ and $93 \%$.
\end{abstract}

Key words: BL Lacertae objects: individual: RGB 2243+203 - galaxies: distances and redshifts

\section{INTRODUCTION}

At high Galactic latitudes, the $\gamma$-ray sky is dominated by active galactic nuclei (AGNs), especially of blazar-type. For example, at high energies (20 MeV - $300 \mathrm{GeV})$, Fermi-LAT has observed 1745 AGNs from which 1718 are associated to either blazars, or blazar candidates (3FGL, Acero et al. 2015). Blazar-type sources are radio-loud AGNs, and under the unification scheme, blazars are AGNs in which the relativistic jets that originate close to the central massive black hole are oriented directly to the observer (Blandford \& Rees 1978). They consist of two kinds of objects: flat-spectrum radio quasars (FSRQs) and BL Lac objects (BLLs). BLLs are distinctly less luminous in $\gamma$-rays than the FSRQs, suggesting that these two types trace probably different accretion regimes (Ghisellini, Maraschi, \& Tavecchio 2009; Ghisellini et al. 2017). While the high luminosity of BLLs al-

^ E-mail: danrosa@inaoep.mx lows their detection at large distances, it also dilutes any spectral feature from its host galaxy (León-Tavares et al. 2011; Furniss et al. 2013; Falomo, Pian, \& Treves 2014; Rosa-González et al. 2017), making the determination of their redshifts very unlikely. At very high energies (VHE; $100 \mathrm{GeV}-100 \mathrm{TeV}$ ) the spectral energy distribution (SED) is strongly affected by the diffuse extra-galactic background light (EBL) and hence appropriate corrections to recover the intrinsic spectral shape must be applied (e.g. Franceschini, Rodighiero, \& Vaccari 2008; Domínguez et al. 2011), before comparing the SED with theoretical emission models (e.g. Dermer \& Schlickeiser 1993; Böttcher et al. 2013). This attenuation significantly reduces the detection volume in the Universe, which is seen in the VHE catalogue (Wakely \& Horan 2008); only 78 extragalactic sources have been detected during the last few decades, 64 being BLLs, 20\% of which have unknown or poorly-constrained redshift. Although VHE blazars are not many, they are very important to understand the most powerful processes in the Universe; 
they are candidates to be the sources of ultra-high energy cosmic rays (Aab et al. 2018), from which only gamma rays and neutrinos may be observed directly (IceCube Collaboration et al. 2018). They are also important for contributing to the estimation of intergalactic magnitudes, like the EBL (Ahnen et al. 2016; H. E. S. S. Collaboration et al. 2017) and intergalactic magnetic field (Caprini \& Gabici 2015; Finke et al. 2015). In all these studies, a precise measurement of the redshift is required.

In recent years several groups have been collecting data to obtain the redshift of the most elusive objects. Shaw et al. (2013) recently compiled the largest sample of $\gamma$-ray selected BLLs with spectroscopic redshifts. They used literature data and their own observations in 4 and 10 meter class telescopes resulting in successful redshift measurements for only $44 \%$ of the 475 studied BLLs. In those cases where the redshift of the targets is immeasurable, Muriel et al. (2015) proposed a statistical method to assess the probability that a BLL belongs to a given system of galaxies. It is generally assumed that BLL nuclei are hosted by luminous elliptical galaxies embedded in groups of galaxies or galaxy clusters (e.g. Falomo, Melnick, \& Tanzi 1990; Muriel 2016; Torres-Zafra et al. 2018), where the nuclear activity is supposed to be enhanced by the interaction with a nearby companion (e.g. Hopkins et al. 2008). The method of Muriel et al. (2015) (slightly modified in Rovero et al. 2016) first requires the spectroscopic identification of a galaxy group in the line-of-sight around the BLL; if a group is identified, then the probability that a given BLL is not a member of that group is given by the probability of finding a BLL in an isolated galaxy times the probability of finding by chance a galaxy group in the line-of-sight of the BLL. If that probability is low enough, the BLL is assumed to be member of the group and the mean redshift of the system is assigned to it. The technique has been used for PKS 0447-439 (Muriel et al. 2015) and PKS 1424+240 (Rovero et al. 2016).

RGB J2243+203 (RGB 2243 hereafter) included in the $\mathrm{TeV}$ catalogue (Wakely \& Horan 2008) was first reported by the $5 \mathrm{GHz}$ survey carried out with the Green Bank telescope (Griffith et al. 1990). It was classified as intermediateenergy-peaked BLL object in Laurent-Muehleisen et al. (1999) and as a high-synchrotron-peaked BLL galaxy in The Third Fermi-LAT Catalog of Sources above $10 \mathrm{GeV}$ (3FHL). The emission of RGB 2243 between $10 \mathrm{GeV}$ and $1 \mathrm{TeV}$ is better fit with a $\log$-parabola function, $\frac{d N}{d E} \propto(E / 16.52 \mathrm{GeV})^{-\Gamma}$, where

$$
\Gamma=(1.767 \pm 0.256)+(0.489 \pm 0.194) \log (E / 16.52 \mathrm{GeV}),
$$

and it has an integrated flux within this band of (1.936 \pm 0.218$) \times 10^{-11} \mathrm{erg} \mathrm{s}^{-1} \mathrm{~cm}^{-2}$ (Ajello et al. 2017).

Sources with hard spectral index and detected by Fermi above $10 \mathrm{GeV}$ are targets of interest for follow up studies at very high energies (VHE; E > $100 \mathrm{GeV}$ ). In fact, RGB 2243 has been detected above $160 \mathrm{GeV}$ by the imaging Cherenkov telescope VERITAS (Abeysekara et al. 2017), and combining their detection with different extragalactic background light (EBL) models they derived a redshift upper limit of $z<1.1$.

Based on optical observations of the RGB 2243 host galaxy, and assuming that it can be treated as a standard candle with $\mathrm{M}_{R}=-22.9 \pm 0.5$, Meisner \& Romani (2010) estimated a redshift lower limit of $z>0.39$. Shaw et al. (2013) established a similar spectroscopic lower limit of $z>0.395$ from intervening absorption systems using the Low Resolution Imaging Spectrograph at the W. M. Keck Observatory. Paiano et al. (2017) obtained a featureless spectrum in the 4100 - $9000 \AA$ wavelength range using long slit observations in the Gran Telescopio Canarias (GTC) ${ }^{1}$. Based also on the assumption that BLL host galaxies are standard candles they proposed a redshift lower limit of $z>0.22$.

In this paper we complement the previous spectroscopic studies by making use of the OSIRIS Multi Object Spectrograph (MOS) mounted on the GTC. We report on the distance and environment of RGB 2243, and also use the method described by Muriel et al. (2015) to statistically associate a redshift to it.

Throughout this paper, we assume a flat cold dark matter cosmology with $\Omega_{\mathrm{M}}=0.3$ and $\mathrm{H}_{0}=70 \mathrm{~km} \mathrm{~s}^{-1} \mathrm{Mpc}^{-1}$.

\section{OBSERVATIONS AND DATA REDUCTION}

The observations were performed using the OSIRIS-MOS installed in the Nasmyth-B focus of the 10.4-m GTC under the program GTC5-15BMEX (PI D. Rosa González). The observations were carried out in service mode, using the R1000R grism. The spectrum is centred at $7430 \AA$ covering the range from 5100 to $10000 \AA$ and a sampling of $2.62 \AA /$ pixel. Using a fixed slit width of $1.2^{\prime \prime}$ we end up with an effective resolution measured on strong sky lines of around $11 \AA$. Targets are located at different positions along the dispersion axis, changing the actual wavelength coverage; the common wavelength range covered by all spectra is 5400-9500 A.

The total observing time was divided in two observing blocks (OB) that were observed on July $11^{\text {th }}$ and $28^{\text {th }}$ in 2016. Each OB consisted of 5 exposures of 790 seconds on target to facilitate removal of cosmic ray hits, and to ensure that the spectrum of the BLL is not saturated. The OBs were accompanied by a set of ancillary files that included observations of Ross 640 as a spectro-photometric standard star, bias, flat-field and arc lamps. Both OBs were observed with air masses lower than 1.1 under clear gray nights and a seeing of between $1^{\prime \prime}$ and $1.2^{\prime \prime}$.

The data reduction was carried out using a new MOS pipeline described in Gómez-González, Mayya, \& RosaGonzález (2016). In short, the code reduces every MOS slit by applying the usual IRAF ${ }^{2}$ scripts for long-slit spectra. To reduce the data, the three different target images were independently corrected by bias. Then we stacked them to obtain a single spectral image where the cosmic rays were successfully removed. After that, every slitlet spectrum was calibrated in wavelength by using as a reference a $\mathrm{He}+\mathrm{Ne}+\mathrm{Ar}$ arc image. The dispersion solution is obtained for every single slitlet and in all cases rms errors lower than $0.4 \AA$ were

1 Gran Telescopio Canarias is a Spanish initiative with the participation of Mexico and the US University of Florida, and is installed at the Roque de los Muchachos in the island of La Palma. 2 IRAF is distributed by the National Optical Astronomy Observatories, which are operated by the Association of Universities for Research in Astronomy, Inc., under cooperative agreement with the National Science Foundation. 


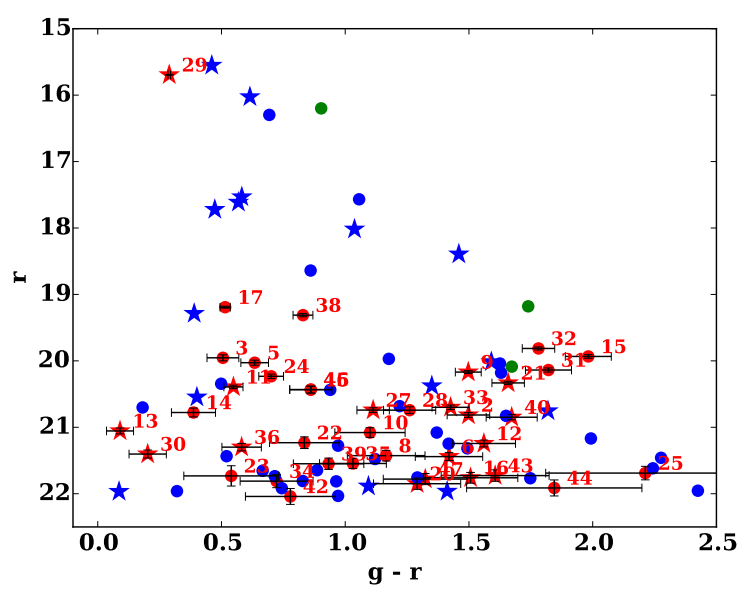

Figure 1. Colour magnitude diagram for all objects brighter than $r \sim 22$ within the MOS field of view. One of the brightest objects corresponds to RGB 2243 (MOS-29) and it is located at the top left corner of the diagram. Objects classified as stars are represented by star symbols, and galaxies by circles. In red are the targets observed by us with the slit number close to the corresponding symbol. Green circles are objects with SDSS spectroscopy. In blue are the objects in the field that we did not observe.

found. This uncertainty produces a systematic error in the redshift calculations of $\sim 10^{-4}$ at a wavelength of $\sim 5500 \AA$.

Finally the image is calibrated in flux by using a sensitivity curve obtained from the observation of Ross 640 . The output of the pipeline is a 2-D spectral image calibrated in both wavelength and flux.

MOS allows us to obtain the spectra of several objects within an effective field-of-view (FOV) of around $7^{\prime} \times 2^{\prime}$. Excluding the stars used as astrometric guides, within the MOS field of view we found 92 targets brighter than $r \sim 22$. We selected our targets based on the SDSS images centred at the position of RGB 2243. The selected FOV contains 168 SDSS objects, and given the nature of our program, where we do not know the redshift of the source, the host galaxy morphological type, or other extra information about the surroundings, we tried to cover as many objects as possible in the OSIRIS FOV taking care of the physical limitations of the mask making procedure, giving preference to those objects classified as galaxies. At the end, we locate the slits on top of 22 objects classified as galaxies and 17 classified as stars. Note that some objects classified as stars could be distant galaxies (including RGB 2243), and in fact objects classified as stars by SDSS, covered by slits 11, 13, 27, 30 and 43 , turned out to be galaxies at different redshifts (Table 1). The colour magnitude diagram (CMD, figure 1) shows the location of the SDSS targets where we differentiate stars from galaxies and we mark those objects selected for spectroscopy. We cover most of the parameter space in the CMD, obtaining good signal to noise spectra for $25 \%$ of the objects brighter than $r=22$.

Figure 2 shows partially the slit positions on top of an $r$-band SDSS image, where we also mark the fiducial stars used for astrometry and areas free of objects used for obtaining reliable sky spectra. The length of the slits goes from $1^{\prime \prime}$ to $10^{\prime \prime}$ and the width is fixed at $1.2^{\prime \prime}$. The SDSS image shows RGB 2243 in slit 29.

The individual spectrum for each object was extracted from the calibrated 2-D spectral image by using the IRAF task apall. In most of the cases the continuum is well detected and a $4^{\text {th }}$ order polynomial function was good enough to fit the trace along the dispersion axis. In the cases where the continuum was not well detected we used the trace solutions found for a nearby object as reference.

In general the extraction window is centred on the peak of the continuum, however for the case of the extraction of the spectrum corresponding to RGB 2243 (MOS-29), and keeping in mind that the emission coming from the centre is expected to be featureless, we choose two apertures that avoid the central pixels. Details of these cuts are given in the next section.

Once the 1-D individual spectra were extracted, we located by eye different spectral features. We looked for the most common lines observed in extra-galactic sources, both in absorption (e.g. Ca II H\&K, Mg band, NaD) and in emission (e.g. hydrogen recombination lines, [OII] $\lambda 3727$, [OIII] $\lambda 5007,[\mathrm{NII}] \lambda 6583)$. Once the lines were identified in a given spectrum we fit a Gaussian profile which returns the position of the peaks (we used the IRAF/splot command stroke $k$ ). The peak position was used to estimate the corresponding redshifts for each individual line. The final redshift is the median of all measured values. The error is given by the standard deviation when we have more than two lines in the spectrum, or the difference between the two redshift values when we have only two lines identified in the spectrum. The systematic error in redshift of $10^{-4}$ due to uncertainties in the wavelength calibration described in Section 2 was added in quadrature. The spectroscopic redshifts and the errors are included in Table 1 . We also included the number of lines used to estimate the redshifts and whether they were found in emission or in absorption. MOS-13, an object classified as a star by the SDSS, is the only target where only one line at $\sim 6042 \AA$ was identified. Its redshift was calculated assuming that the observed broad emission line corresponds to $\mathrm{Mg}$ II $\lambda 2797$.

\section{RESULTS}

\subsection{Spectrum of RGB 2243}

The flux calibrated spectrum of the RGB 2243 galaxy is plotted in Figure 3 (Top). The spectrum was barely smoothed by using a Savitzky-Golay filter (e.g. Press et al. 1989), with a window size of 3 pixels and a one-degree polynomial. ${ }^{3}$

The spectrum corresponds to the entire BLL spectrum including the central pixels where the featureless continuum has its maximum contribution. It was extracted using an aperture of 8 pixels $\left(2^{\prime \prime}\right)$, and it has a signal to noise ratio $(\mathrm{S} / \mathrm{N})$ of $\sim 130$ which is about two times worse than the one published by Shaw et al. (2013) based on observations

3 The Savitzky-Golay filter is a particular type of low-pass filter. We make use of the routine provided by the SciPy organization at SciPy.org. Infor- mation of the algorithm and multiple references can be found in the SciPy pages. 


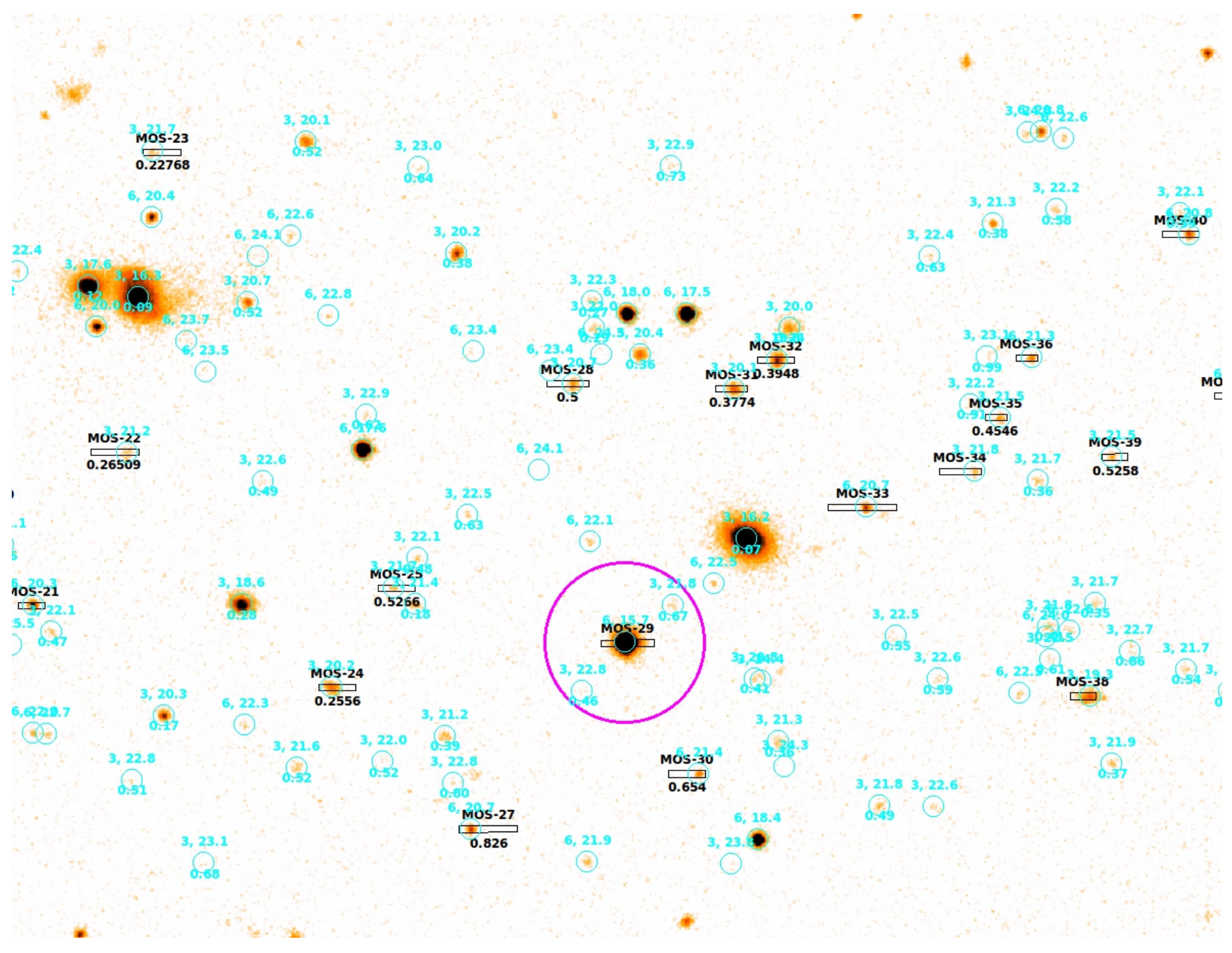

Figure 2. $r$-band SDSS image of a FOV of $3.7^{\prime} \times 2.8^{\prime}$ showing the position of the MOS slits (black rectangles). Above the slits are the slit numbers and the spectroscopic redshifts are indicated below them (see Table 1). Slit 29 marks the position of RGB 2243, and it is enclosed by a purple circle of $15^{\prime \prime}$ in radius. Cyan circles show the positions of SDSS objects; SDSS classification (3 for galaxies, 6 for stars) and the $r$ magnitude are shown above each one. Below we add the photometric redshift if any. North is at the top, East to the left, and the image covers partially the OSIRIS FOV.

with the Low Resolution Imaging Spectrograph at the W. M. Keck Observatory, and within the range of $\mathrm{S} / \mathrm{N}$ values presented recently by Paiano et al. (2017) obtained with the GTC. We also show the position of the sodium line and several telluric absorption lines.

In our previous work where we examined the spectrum of the BLL 3FGL J0909.0+2310, we noticed that avoiding the central part of the continuum emission facilitates the detection of absorption spectral features with better signal to noise ratio (Rosa-González et al. 2017). Following this result we realized two different cuts which avoid the featureless continuum. One corresponds to an extraction window centred 2 pixels to the west of the continuum peak, and the other to a centre located 2 pixels to the east. Both cuts were performed using an extraction window of 4 pixels $\left(1^{\prime \prime}\right)$. We did not find any spectral feature in any of the three spectra analyzed.

\subsection{Redshift of Galaxies in the OSIRIS FOV}

We obtain spectroscopic redshifts for 22 of the 39 observed targets. Targets for which we cannot extract the redshift include objects close to the slit borders and those where the signal to noise ratio was too low to obtain any spectral feature. Table 1 lists the SDSS properties of the selected targets, and the spectroscopic redshifts.

We notice that, additionally to our observations, three galaxies within the observed FOV have SDSS spectroscopy and they are included in the data release 12 (Alam et al. 2015). We include in table 2 the position and spectroscopic redshifts of these sources.

To study the existence of galaxy groups within the OSIRIS FOV we create a histogram showing the redshift distribution based on photometric and spectroscopic redshifts (figure 4). The spectroscopic data come from our MOS observations and the 3 galaxies from SDSS. The bin of the spectroscopic redshift histogram is of 0.0075 , and corre- 


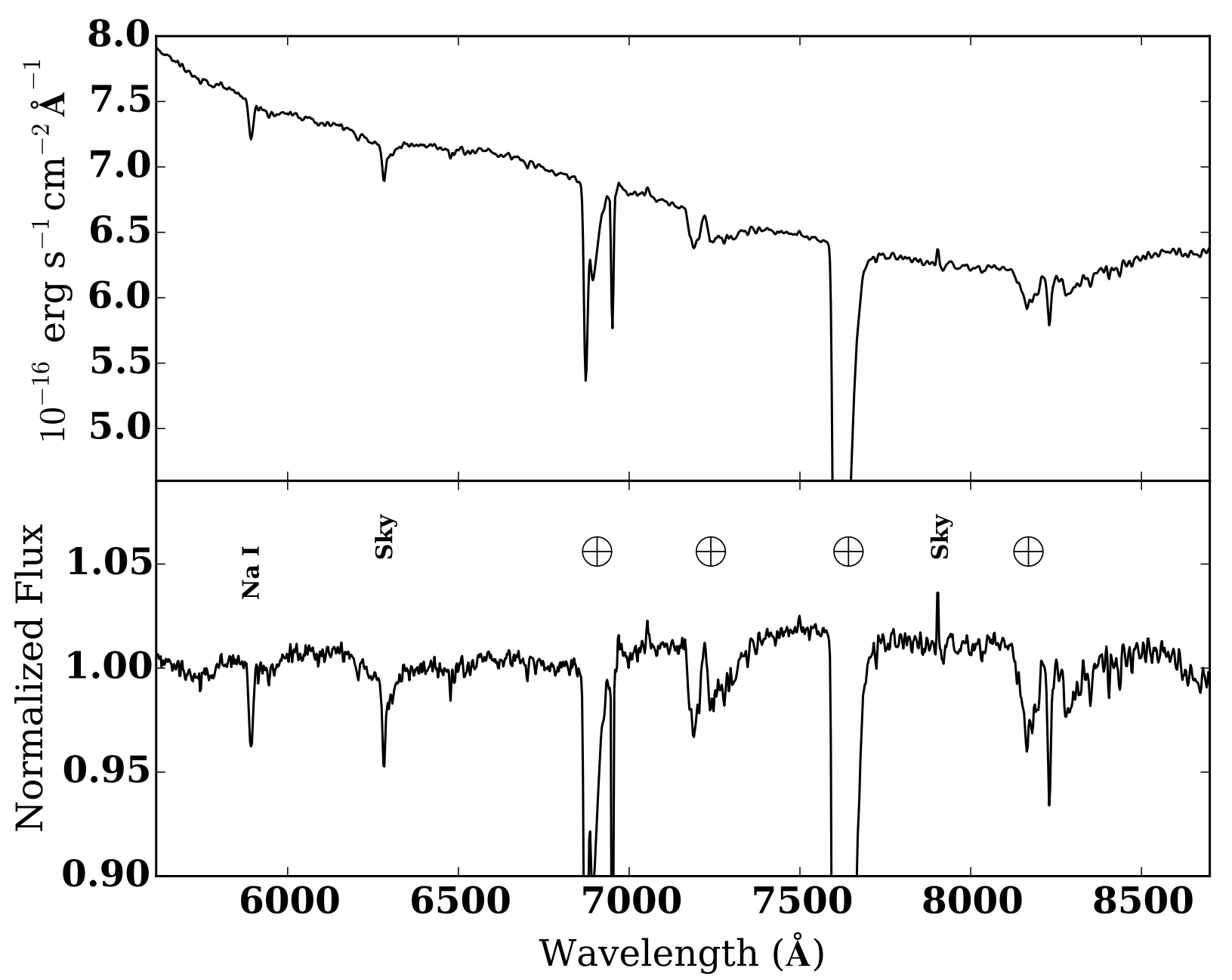

Figure 3. The observed sky-subtracted spectrum before (top panel) and after normalization by a smooth continuum (bottom panel). All the absorption/emission features seen in the spectra are either due to telluric absorption lines $(\bigoplus)$ or residual sky/street lamp lines ([OI]6300.304, Na I etc.). Only a section of the full OSIRIS spectral coverage is plotted. The observed bump at $8500 \AA$ is due to the $2^{\text {nd }}$ order contribution, that could not be properly corrected with the observations of the standard star, due to the extremely blue spectrum of the BL Lac.

sponds to a velocity of around $2000 \mathrm{~km} \mathrm{~s}^{-1}$. Values this large have been found in the study of the velocity dispersion of massive galaxy clusters and is ideal for our blind search of groups. The photometric redshifts were obtained from the SDSS data release 12 (Beck et al. 2016) which includes all the galaxy-type objects within the OSIRIS FOV $\left(7.4^{\prime} \times 2.3^{\prime}\right.$ centered in RGB 2243). The bin size of the photometric redshift distribution was fixed in 0.08 which corresponds to the median of the photometric redshift errors of the selected galaxies.

A small peak with four members, appears in the spectroscopic distributions at redshift 0.53. As mentioned in the introduction BLL are believed to be hosted by elliptical galaxies (Muriel 2016) and are primarily found in groups of three or more members with typical virial radius of around 1 Mpc (Merchán \& Zandivarez 2002). In fact, three of the four galaxies with redshift around 0.53 show the typical absorption lines observed in local elliptical galaxies (figure 5). The possibility that RGB 2243 belongs to that group at $z \sim 0.53$ is explored further in the next section.

\subsection{Statistical Redshift}

As seen in the previous section, the redshift distribution (figure 4) in the line of sight of RGB 2243 shows the presence of a group of galaxies with 4 spectroscopic members. It is also observed that the group could be more numerous if we take into account the four galaxies with photometric redshift at the same distance. The relative position of these two groups of galaxies with respect to RGB 2243 are plotted in figure 6 , and different properties of them are presented in tables 1 , and 3 .

Using only the four galaxies with spectroscopic redshift, we computed the mean redshift $\left(z_{\text {mean }}\right)$ and the velocity dispersion $\left(\sigma_{v}\right)$ using the Gapper estimator defined by Beers, Flynn, \& Gebhardt (1990) and the virial radius $\left(R_{\text {vir }}\right)$ following Nurmi et al. (2013). We obtained the following values: 


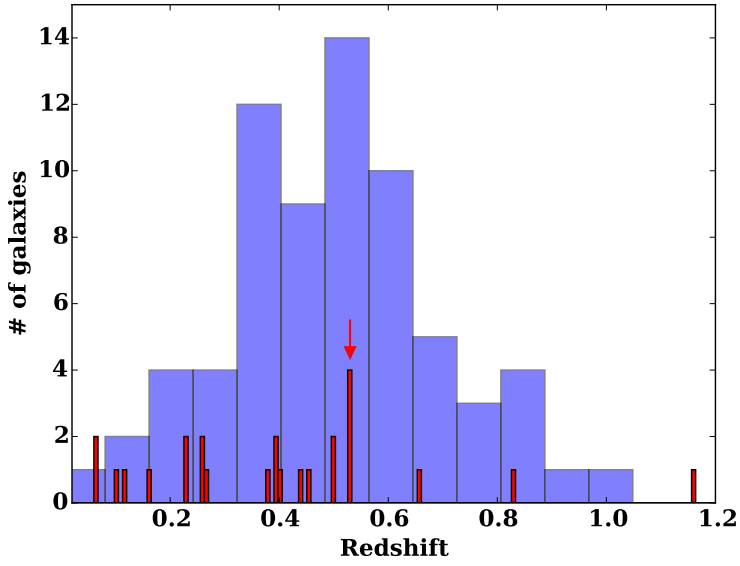

Figure 4. Spectroscopic (red) and photometric (blue) redshift distributions of the targets within the OSIRIS FOV. The bin size of the spectroscopic distribution is 0.0075 , and 0.08 for the photometric distribution. One small group of galaxies with spectroscopic redshifts around 0.53 is identified and highlighted with the red arrow. The red histogram includes the three objects with SDSS spectroscopy.

$z_{\text {mean }}=0.527 \pm 0.001 ; R_{\text {vir }}=0.84 \mathrm{Mpc}\left(2.2^{\prime}\right.$ at that distance $)$ and $\sigma_{v}=197 \mathrm{~km} \mathrm{~s}^{-1} \pm 124$. Given that the mean redshift and the velocity dispersion are computed using only four galaxies, the quoted values are susceptible to errors associated with the statistics of small numbers and should be taken with caution.

We follow the procedure described in Muriel et al. (2015) and Rovero et al. (2016) to estimate the probability that RGB 2243 is a member of the group of galaxies at $z=0.527$ described by the parameters calculated previously. In short the method can be described in the following steps, i) we use the most complete inner field of the zCOSMOS $20 \mathrm{k}$ group catalog (Knobel et al. 2012, hereafter 20k catalog), which has 1498 identified groups; ii) we test the completeness of our sample of spectroscopic redshifts computing the fraction of galaxies per bin of apparent magnitude that have spectroscopic redshift; iii) we extract from the 20k catalog a random subsample of galaxies (a pruned catalog) by selecting galaxies that have approximately the same magnitude distribution as our spectroscopic sample. In this process we take into account that the mean spectroscopic completeness of zCOSMOS is $56 \%$; iv) the pruned catalog of galaxies is used to define a catalog of groups that includes those systems that survive the random selection of galaxies (the new richness of groups is also computed); v) within the pruned catalog of groups of galaxies, we select random positions and see the coincidence with groups with four or more members; vi) we repeat the whole procedure 100 times and compute the mean probability to find by chance a group of galaxies like the observed one. We found a probability of $22.5 \pm 2.0 \%$ of finding a group of four or more members in an observation similar to the one reported here. The error corresponds to the standard deviation of the 100 realizations.

If the group of galaxies found in the line of sight does not host the BLL, it means that the host galaxy is an isolated object. As it was pointed out, Muriel (2016) found that

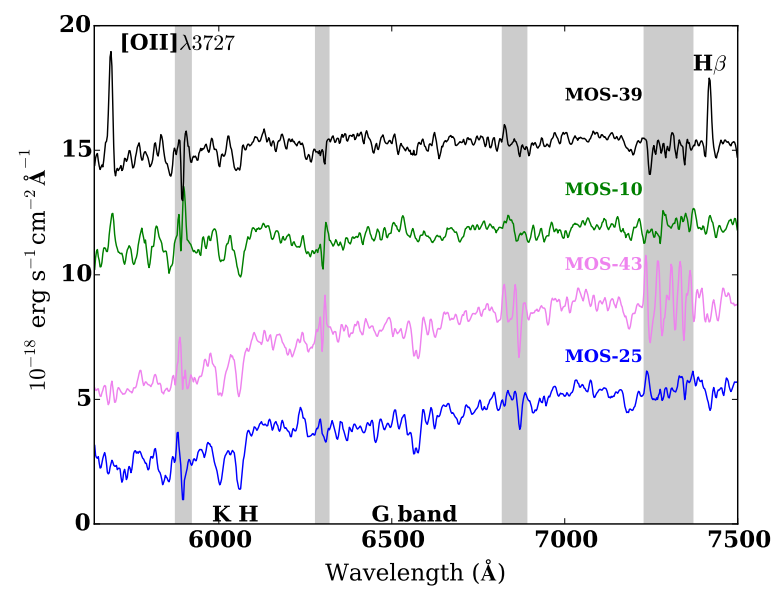

Figure 5. GTC spectra of the galaxies with spectroscopic redshift at redshift around 0.53 . The position of common absorption and emission lines are marked. The gray vertical bands show the position of strong sky lines where the sky substraction is far from perfect and fake structures appear in the spectra. From the bottom to the top the spectra have been artificially shifted by adding constant continuum values of 4,8 and $12 \times 10^{-18} \mathrm{erg} \mathrm{s}^{-1} \mathrm{~cm}^{-2} \AA^{-1}$. The spectra were filtered by using a Savitzky-Golay filter with a window size of 3 pixels and a polynomial of order one.

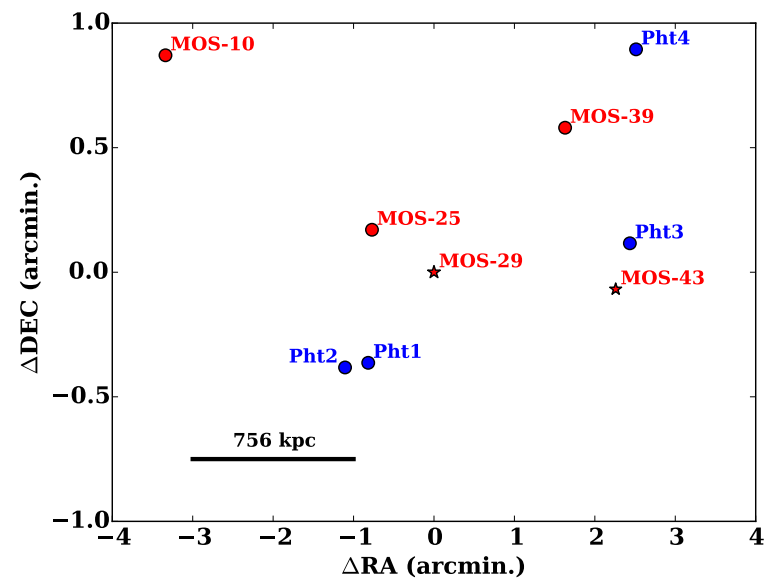

Figure 6. Relative position of the galaxies with redshits around 0.53. The diagram is centred in the position of MOSS-29 (RGB 2243) and we plotted in red the galaxies with spectroscopic redshift and in blue the ones with photometric redshifts. Both MOS-29 and MOS-43 were classified as stars by the SDSS, and we use a star-symbol to distinguish them. The physical scale is displayed in the bottom corner of the image.

at least $67 \pm 8 \%$ of the BLLs are in galaxies that are members of groups or clusters of galaxies. Since the probability of the BLL to be hosted by an isolated galaxy is independent of the probability computed above of finding a group by chance, then the probability of RGB 2243 not being associated with the group of four members is the joint probability of having both, a group by chance $(22.5 \pm 2.0 \%)$ and an isolated BLL $(\lesssim 33 \pm 8 \%)$ is $\lesssim 7.4 \pm 1.8 \%$ (the error is computed 
using propagation of uncertainties). This implies that - if we assumed that the BL Lac is an isolated galaxy - the possible membership of RGB 2243 to the group of galaxies found at $\mathrm{z}=0.527$ has an occurrence probability of $\sim 92.6 \pm 1.8 \%$. On the other hand the joint probability of finding a group with five members and the BL Lac belonging to an undetected group of galaxies is of $14 \pm 2 \%$, and therefore the probability of RGB 2243 being a member of the group at $\mathrm{z}=0.527$ would be of $86 \pm 2 \%$.

\section{CONCLUSIONS}

We have presented high signal to noise optical spectrum of the elusive object RGB 2243. The spectrum does not show any spectral features that can be used to determine its distance with precision. Based on the spectroscopic observations of the BLL surroundings we found four galaxies at a redshift of around 0.53 . Three of them have the typical absorption lines studied in local elliptical galaxies. By using a statistical analysis where we take into account that BLL objects are usually found in galaxy groups we found that, if we use only the spectroscopic confirmed object at redshift $\sim 0.53$, then the probability that RGB 2243 would be a member of that group is between $86 \%$ and $93 \%$. Based on these results we propose that RGB $2243+203$ is hosted by a galaxy for which we estimate an statistical redshift of $z=0.527 \pm 0.001$. Being a member of that group is in agreement with the redshift upper limits proposed by the EBL studies reported by the VERITAS team Abeysekara et al. (2017) and the lower limits obtained by recent optical observations (Shaw et al. 2013; Paiano et al. 2017).

\section{ACKNOWLEDGEMENTS}

We thank the support team at GTC and the anonymous referee for helpful suggestions. Based on observations made with the Gran Telescopio Canarias (GTC), installed at the Spanish Observatorio del Roque de los Muchachos of the Instituto de Astrofísica de Canarias, in the island of La Palma. This work is partly financed by CONACyT - the Mexican research Council - research grants CB-2010-01-155142G3 (PI:YDM) and CB-2011-01-167281-F3 (PI:DRG). SCL thanks CONACyT for her studentship. This paper has been partially supported with grants from CONICET - the Argentinian research Council - and SeCyT, Universidad Nacional de Córdoba, Argentina.

Funding for the SDSS and SDSS-II has been provided by the Alfred P. Sloan Foundation, the Participating Institutions, the National Science Foundation, the U.S. Department of Energy, the National Aeronautics and Space Administration, the Japanese Monbukagakusho, the Max Planck Society, and the Higher Education Funding Council for England. The SDSS Web Site is http://www.sdss.org/.

The SDSS is managed by the Astrophysical Research Consortium for the Participating Institutions. The Participating Institutions are the American Museum of Natural History, Astrophysical Institute Potsdam, University of Basel, University of Cambridge, Case Western Reserve University, University of Chicago, Drexel University, Fermilab, the Institute for Advanced Study, the Japan Participation
Group, Johns Hopkins University, the Joint Institute for Nuclear Astrophysics, the Kavli Institute for Particle Astrophysics and Cosmology, the Korean Scientist Group, the Chinese Academy of Sciences (LAMOST), Los Alamos National Laboratory, the Max-Planck-Institute for Astronomy (MPIA), the Max-Planck-Institute for Astrophysics (MPA), New Mexico State University, Ohio State University, University of Pittsburgh, University of Portsmouth, Princeton University, the United States Naval Observatory, and the University of Washington.

\section{REFERENCES}

Aab A., et al., 2018, ApJ, 853, L29

Abeysekara A. U., et al., 2017, ApJSS, 233, 1

Acero F., et al., 2015, ApJS, 218, 23

Ahnen M. L., et al., 2016, A\&A, 590, A24

Ajello M., et al., 2017, ApJS, 232, 18

Alam S., et al., 2015, ApJS, 219, 12

Allen J. T., Hewett P. C., Maddox N., Richards G. T., Belokurov V., 2011, MNRAS, 410, 860

Beck R., Dobos L., Budavári T., Szalay A. S., Csabai I., 2016, MNRAS, 460, 1371

Beers T. C., Flynn K., Gebhardt K., 1990, AJ, 100, 32

Blandford R. D., Rees M. J., 1978, PhyS, 17, 265

Böttcher M., Reimer A., Sweeney K., Prakash A., 2013, ApJ, 768, 54

Caprini C., Gabici S., 2015, PhRvD, 91, 123514

Dermer C. D., Schlickeiser R., 1993, ApJ, 416, 458

Domínguez A., et al., 2011, MNRAS, 410, 2556

Falomo R., Melnick J., Tanzi E. G., 1990, Natur, 345, 692

Falomo R., 1996, MNRAS, 283, 241

Falomo R., Pian E., Treves A., 2014, A\&ARv, 22, 73

Finke J. D., Reyes L. C., Georganopoulos M., Reynolds K., Ajello M., Fegan S. J., McCann K., 2015, ApJ, 814, 20

Franceschini A., Rodighiero G., Vaccari M., 2008, A\&A, 487, 837

Furniss A., Fumagalli M., Danforth C., Williams D. A., Prochaska J. X., 2013, ApJ, 766, 35

Ghisellini G., Maraschi L., Tavecchio F., 2009, MNRAS, 396, L105

Ghisellini G., Righi C., Costamante L., Tavecchio F., 2017, MNRAS, 469, 255

Gómez-Gónzalez V. M. A., Mayya Y. D., Rosa-González D., 2016, MNRAS, 460, 1555

Griffith M., Langston G., Heflin M., Conner S., Lehar J., Burke B., 1990, ApJS, 74, 129

H. E. S. S. Collaboration, et al., 2017, A\&A, 606, A59

Hopkins P. F., Hernquist L., Cox T. J., Kereš D., 2008, ApJS, 175, 356-389

IceCube Collaboration, et al., 2018, Sci, 361, eaat1378

Knobel C., et al., 2012, ApJ, 753, 121

Koulouridis E., Plionis M., Chavushyan V., Dultzin D., Krongold Y., Georgantopoulos I., León-Tavares J., 2013, A\&A, 552, A135

Laurent-Muehleisen S. A., Kollgaard R. I., Feigelson E. D., Brinkmann W., Siebert J., 1999, ApJ, 525, 127

León-Tavares J., Valtaoja E., Chavushyan V. H., Tornikoski M., Añorve C., Nieppola E., Lähteenmäki A., 2011, MNRAS, 411, 1127

Meisner A. M., Romani R. W., 2010, ApJ, 712, 14

Merchán M., Zandivarez A., 2002, MNRAS, 335, 216

Muriel H., Donzelli C., Rovero A. C., Pichel A., 2015, A\&A, 574, A101

Muriel H., 2016, A\&A, 591, L4

Nolan P. L., et al., 2012, ApJS, 199, 31

Nurmi P., et al., 2013, MNRAS, 436, 380 
Table 1. Targets observed with GTC-MOS. Columns 1 to 6 give: slit number (ID), SDSS coordinates, type (3 for galaxies, 6 for stars), SDSS $r$ band, and SDSS $g-r$ colour. The spectroscopic redshift $\left(z_{\mathrm{sp}}\right)$, its error and the number of lines (N) used for the redshift determination are given in columns 7 and 8 . Column 8 also indicates whether the lines were observed in absorption (A) or in emission (E). Column 9 indicates the projected and physical distance to RGB 2243, for those galaxies with spectroscopic redshift around 0.53. The slits on fiducial stars or those used for sky measurements are not shown.

\begin{tabular}{|c|c|c|c|c|c|c|c|c|c|c|}
\hline ID & $\mathrm{RA}\left({ }^{\circ}\right)$ & $\operatorname{DEC}\left({ }^{\circ}\right)$ & Type & $r$ (mag.) & $g-r$ (mag.) & $z_{\mathrm{sp}}$ & $\mathrm{N}$ & \multicolumn{2}{|c|}{$\begin{array}{c}\text { Distances } \\
\text { (arcmin. \& Mpc) }\end{array}$} & Comments \\
\hline MOS-2 & 341.05611 & 20.35854 & 6 & $20.81 \pm 0.04$ & $1.50 \pm 0.09$ & - & - & & & (b) \\
\hline MOS-3 & 341.05403 & 20.36081 & 3 & $19.95 \pm 0.05$ & $0.51 \pm 0.06$ & $0.0625 \pm 0.0002$ & $5 / \mathrm{E}$ & & & \\
\hline MOS-5 & 341.04905 & 20.33917 & 3 & $20.03 \pm 0.04$ & $0.63 \pm 0.06$ & $0.1137 \pm 0.0001$ & $4 / \mathrm{E}$ & & & \\
\hline MOS-6 & 341.04592 & 20.35365 & 6 & $21.44 \pm 0.06$ & $1.42 \pm 0.14$ & - & - & & & (b) \\
\hline MOS-8 & 341.03945 & 20.36384 & 3 & $21.43 \pm 0.08$ & $1.17 \pm 0.16$ & $0.2272 \pm 0.0001$ & $4 / \mathrm{E}$ & & & \\
\hline MOS-9 & 341.03655 & 20.35353 & 6 & $20.17 \pm 0.02$ & $1.50 \pm 0.05$ & - & - & & & (b) \\
\hline MOS-10 & 341.03362 & 20.36537 & 3 & $21.08 \pm 0.07$ & $1.10 \pm 0.14$ & $0.5280 \pm 0.0010$ & $3 / \mathrm{A}$ & 3.4 & 1.3 & (e) \\
\hline MOS-11 & 341.03178 & 20.34478 & 6 & $20.39 \pm 0.03$ & $0.55 \pm 0.04$ & $0.4410 \pm 0.0020$ & $4 / \mathrm{A}$ & & & \\
\hline MOS-12 & 341.02989 & 20.35458 & 6 & $21.24 \pm 0.05$ & $1.56 \pm 0.13$ & - & - & & & (b) \\
\hline MOS-13 & 341.02779 & 20.37159 & 6 & $21.05 \pm 0.04$ & $0.09 \pm 0.05$ & 1.1590 & $1 / \mathrm{E}$ & & & (d) \\
\hline MOS-14 & 341.02600 & 20.37331 & 3 & $20.78 \pm 0.07$ & $0.39 \pm 0.09$ & $0.0985 \pm 0.0001$ & $4 / \mathrm{E}$ & & & \\
\hline MOS-15 & 341.02449 & 20.34902 & 3 & $19.93 \pm 0.03$ & $1.98 \pm 0.09$ & $0.4000 \pm 0.0008$ & $6 / \mathrm{A}$ & & & \\
\hline MOS-16 & 341.02261 & 20.34985 & 6 & $21.76 \pm 0.08$ & $1.51 \pm 0.19$ & - & - & & & (b) \\
\hline MOS-17 & 341.02033 & 20.35083 & 3 & $19.19 \pm 0.01$ & $0.51 \pm 0.02$ & $0.1600 \pm 0.0100$ & $4 / \mathrm{E}$ & & & \\
\hline MOS-20 & 341.01367 & 20.35787 & 6 & $21.85 \pm 0.09$ & $1.29 \pm 0.18$ & - & - & & & (b) \\
\hline MOS-21 & 341.01118 & 20.35279 & 6 & $20.33 \pm 0.03$ & $1.66 \pm 0.07$ & - & - & & & (b) \\
\hline MOS-22 & 341.00660 & 20.36087 & 3 & $21.23 \pm 0.09$ & $0.83 \pm 0.14$ & $0.2651 \pm 0.0001$ & $4 / \mathrm{E}$ & & & \\
\hline MOS-23 & 341.00392 & 20.37651 & 3 & $21.73 \pm 0.15$ & $0.54 \pm 0.19$ & $0.2277 \pm 0.0001$ & $4 / \mathrm{E}$ & & & \\
\hline MOS-24 & 340.99415 & 20.34854 & 3 & $20.23 \pm 0.03$ & $0.70 \pm 0.05$ & $0.2556 \pm 0.0001$ & $8 / \mathrm{E}$ & & & \\
\hline MOS-25 & 340.99083 & 20.35370 & 3 & $21.69 \pm 0.10$ & $2.21 \pm 0.40$ & $0.5266 \pm 0.0007$ & $5 / \mathrm{A}$ & 0.8 & 0.3 & (e) \\
\hline MOS-27 & 340.98573 & 20.34112 & 6 & $20.74 \pm 0.04$ & $1.11 \pm 0.07$ & $0.8260 \pm 0.0020$ & $3 / \mathrm{A}$ & & & \\
\hline MOS-28 & 340.98131 & 20.36440 & 3 & $20.74 \pm 0.05$ & $1.26 \pm 0.11$ & $0.5000 \pm 0.0004$ & $2 / \mathrm{A}$ & & & \\
\hline MOS-29 & 340.97798 & 20.35086 & 6 & $15.69 \pm 0.00$ & $0.29 \pm 0.01$ & - & - & & & (a) \\
\hline MOS-30 & 340.97466 & 20.34401 & 6 & $21.40 \pm 0.06$ & $0.20 \pm 0.08$ & $0.6540 \pm 0.0020$ & $2 / \mathrm{A}$ & & & \\
\hline MOS-31 & 340.97217 & 20.36413 & 3 & $20.14 \pm 0.03$ & $1.82 \pm 0.09$ & $0.3774 \pm 0.0003$ & $6 / \mathrm{A}$ & & & \\
\hline MOS-32 & 340.96972 & 20.36563 & 3 & $19.81 \pm 0.02$ & $1.78 \pm 0.07$ & $0.3948 \pm 0.0005$ & $6 / \mathrm{A}$ & & & \\
\hline MOS-33 & 340.96487 & 20.35790 & 6 & $20.70 \pm 0.03$ & $1.43 \pm 0.08$ & - & - & & & (b) \\
\hline MOS-34 & 340.95978 & 20.35983 & 3 & $21.81 \pm 0.10$ & $0.72 \pm 0.15$ & - & - & & & (c) \\
\hline MOS-35 & 340.95752 & 20.36262 & 3 & $21.54 \pm 0.08$ & $1.03 \pm 0.13$ & $0.4546 \pm 0.0002$ & $3 / \mathrm{E}$ & & & \\
\hline MOS-36 & 340.95587 & 20.36576 & 6 & $21.30 \pm 0.06$ & $0.58 \pm 0.08$ & - & - & & & (c) \\
\hline MOS-38 & 340.95286 & 20.34806 & 3 & $19.31 \pm 0.02$ & $0.83 \pm 0.04$ & - & - & & & (c) \\
\hline MOS-39 & 340.95081 & 20.36053 & 3 & $21.55 \pm 0.09$ & $0.93 \pm 0.14$ & $0.5258 \pm 0.0007$ & $3 / \mathrm{E}$ & 1.7 & 0.7 & (e) \\
\hline MOS-40 & 340.94721 & 20.37217 & 6 & $20.85 \pm 0.04$ & $1.67 \pm 0.10$ & - & - & & & (b) \\
\hline MOS-42 & 340.94307 & 20.36790 & 3 & $22.04 \pm 0.12$ & $0.78 \pm 0.18$ & - & - & & & (c) \\
\hline MOS-43 & 340.94033 & 20.34972 & 6 & $21.73 \pm 0.09$ & $1.61 \pm 0.22$ & $0.5268 \pm 0.0002$ & $3 / \mathrm{A}$ & 2.3 & 0.9 & (e) \\
\hline MOS-44 & 340.93744 & 20.35147 & 3 & $21.91 \pm 0.12$ & $1.84 \pm 0.35$ & - & - & & & (c) \\
\hline MOS-45 & 340.93381 & 20.37821 & 3 & $20.43 \pm 0.05$ & $0.86 \pm 0.09$ & $0.2573 \pm 0.0006$ & $4 / \mathrm{E}$ & & & \\
\hline MOS-46 & 340.93113 & 20.37710 & 3 & $20.43 \pm 0.05$ & $0.86 \pm 0.09$ & - & - & & & (c) \\
\hline MOS-47 & 340.92678 & 20.37309 & 6 & $21.78 \pm 0.08$ & $1.32 \pm 0.17$ & - & - & & & (b) \\
\hline
\end{tabular}

(a) Main target, (b) field star, (c) no spectral features detected, (d) the reported $z=1.1590$ assume that the detected line correspond to Mg II $\lambda 2797$, and (e) galaxies with spectroscopic redshift around 0.53.

Table 2. Objects within the FOV and SDSS spectroscopy.

\begin{tabular}{ccc}
\hline $\mathrm{RA}\left({ }^{\circ}\right)$ & $\mathrm{DEC}\left({ }^{\circ}\right)$ & $z_{\mathrm{sp}}$ \\
\hline 340.93366 & 20.34935 & $0.37943 \pm 0.00008$ \\
340.97133 & 20.35645 & $0.06514 \pm 0.00001$ \\
340.99589 & 20.37720 & $0.50023 \pm 0.00011$ \\
\hline
\end{tabular}

Paiano S., Landoni M., Falomo R., Treves A., Scarpa R., Righi C., 2017, ApJ, 837, 144

Press W. H., Flannery B. P., Teukolsky S. A., Vetterling W. T., 1989, in Numerical Recipes in C. The Art of Scientific Computing (Cambridge Univ. Pres)

Rosa-González D., et al., 2017, MNRAS, 466, 540
Rovero A. C., Muriel H., Donzelli C., Pichel A., 2016, A\&A, 589, A92

Shaw M. S., et al., 2013, ApJ, 764, 135

Torres-Zafra J., Cellone S. A., Buzzoni A., Andruchow I., Portilla J. G., 2018, MNRAS, 474, 3162

Wakely, S. P., Horan, D., 2008, Proceedings of the 30th International Cosmic Ray Conference. July 3 - 11, 2007, Mérida, Yucatán, Mexico. Edited by R. Caballero, et al. Universidad Nacional Autónoma de México, Mexico City, Mexico, 2008. Volume 3, p.1341-1344

This paper has been typeset from a $\mathrm{T}_{\mathrm{E}} \mathrm{X} / \mathrm{LAT}_{\mathrm{E}} \mathrm{X}$ file prepared by the author. 
Table 3. Properties of the SDSS galaxies with photometric redshift around 0.53. The columns show the position, r-magnitude, colors, photometric redshift and distances to RGB 2243.

\begin{tabular}{cccccccc}
\hline ID & $\mathrm{RA}\left({ }^{\circ}\right)$ & DEC $\left(^{\circ}\right)$ & $r$ (mag.) & $g-r$ (mag.) & $z_{\text {ph }}$ & \multicolumn{2}{c}{$\begin{array}{c}\text { Distance } \\
\text { (arcmin. \& Mpc) }\end{array}$} \\
\hline Pht-1 & 340.99161 & 20.344800 & $21.96 \pm 0.21$ & $0.32 \pm 0.26$ & $0.520192 \pm 0.191746$ & 0.9 & 0.3 \\
Pht-2 & 340.99641 & 20.344489 & $21.65 \pm 0.10$ & $0.89 \pm 0.17$ & $0.520293 \pm 0.137049$ & 1.2 & 0.4 \\
Pht-3 & 340.93739 & 20.352797 & $24.14 \pm 0.62$ & $-1.03 \pm 0.65$ & $0.523156 \pm 0.217098$ & 2.4 & 0.9 \\
Pht-4 & 340.93611 & 20.365772 & $22.26 \pm 0.15$ & $1.22 \pm 0.30$ & $0.519344 \pm 0.062896$ & 2.7 & 1.0 \\
\hline
\end{tabular}

